\title{
Validação ou tradução? Questionários de avaliação de qualidade de vida em psoríase
}

O artigo "Validação de questionários de avaliação de qualidade de vida em pacientes de psoríase" detalha a tradução daqueles questionários. A definição do termo validação para estudos epidemiológicos e clínicos correlacionase com a significância do dado avaliado. ${ }^{1.5}$ A validação requer a comparação do dado com uma medida objetiva, idealmente um teste padrão ouro; o que se torna difícil para qualidade de vida. Nesse caso, é possível avaliar a validação preditiva correlacionando-a com a terapêutica. A tradução do questionário do Professor Finlay está disponível desde sua idealização na página da internet da Universidade de Walles (www.ukdermatology.co.uk/quality/pdiquest.asp).

Tendo aplicado esse questionário inúmeras vezes em pacientes envolvidos em pesquisas clínicas do Serviço de Dermatologia da Universidade Federal do Paraná, salientamos dois aspectos importantes. O primeiro, é a dificuldade dos pacientes em quantificar "muitíssimo" (very much) e "muito" (a lot), talvez pela pouca utilização do termo muitíssimo em nossa linguagem diária. Sugerimos a seguinte graduação (em discussão com o autor do questionário): muito (em vez de muitíssimo), médio (em vez de muito), pouco e nada. Um segundo aspecto é a dúvida gerada na primeira pergunta do questionário, quanto a atividades dentro e fora de casa, incluindo dois comportamentos numa mesma questão, não só dentro de casa e no jardim como na questão original (around the house or garden).

O processo de validação de um questionário para uma determinada língua é demorado e caro. Sem dúvida existe a necessidade de validação desses questionários para o português; entretanto é preciso envolver pacientes de diversas regiões, classes sociais e graus de instrução nessa avaliação. Pequenas alterações podem ser realizadas e discutidas com os autores para melhor reprodutibilidade dos dados em populações variadas.

\section{REFERÊNCIAS}

1. Kilpeläimen M, Terho EO, Helenius H, Koskenvuo M. Validation of a new questionnaire on asthma, allergic rhinitis and conjunctivitis in young adults. Allergy. 2001; 56:377-84.

2. Kruizenga HM, Seidell JC, de Vet HC, Wierdsma NJ, van Bokhorst-de van der Schueren MA. Development and validation of a hospital screening tool for malnutrition: the short nutritional assessment questionnaire (SNAQ((c))). Clin Nutr. $2005 ; 24: 75-82$.

3. Yusuf SA, Jorge JM, Habr-Gama A, Kiss DR, Rodrigues JG. Evaluation of quality of life in anal incontinence: validation of the questionnaire FIQL (Fecal Incontinence Quality of Life). Arq Gastroenterol. 2004; 41:202-8.

4. Cummings SR, Stewart AL, Hulley SB. Designing Questionaires and Data Collection Instruments. In: Hulley SB, Cummings SR, Browner WS, Grady D, Hearst N, Newmen TB. Designing Clinical Research: an epidemiological aproach. Philadelphia: Lipincott; 2001. p.231-46.

5. Browner WS. Methods. In: Browner WS. Publishing and presenting clinical research. Baltimore: Lipincott; 1999. p.25-40.

Fabiane Mulinari-Brenner

Médica Dermatologista; Mestre em Medicina Interna; Professora Assistente em Dermatologia; Universidade Federal do Paraná (PR).

Hermênio Cavalcante Lima

Médico Dermatologista e Alergista; Doutor em Imunologia; Professor Adjunto de Patologia Médica; Universidade Federal do Paraná (PR).

ENDEREÇO PARA CORRESPONDENCIA:

Fabiane Mulinari-Brenner

Rua Chichorro Jr, 144 - 131 - Cabral

Curitiba PR 80035-040

Tel./Fax: 41-2643755

E-mail:fmbrenner@uol.com.br 